\title{
An approach for estimation of optimal energy flows in battery storage devices for electric vehicles in the smart grid
}

\author{
Gergana Vacheva ${ }^{1, *}$, Hristiyan Kanchev ${ }^{1}$, Nikolay Hinov ${ }^{1}$ and Rad Stanev ${ }^{2}$ \\ ${ }^{1}$ Technical University of Sofia, Department of Power Electronic, Sofia, 1000, bul. Kl. Ohridsky 8, Bulgaria \\ ${ }^{2}$ Technical University of Sofia, Electrical Power Engineering Department, Sofia, 1000, bul. K1. Ohridsky 8, Bulgaria
}

\begin{abstract}
While the number of the vehicle actuated with liquid fuels are settled, the count of electric vehicles is increasing. For the present moment most of them are scheduled for daily urban usage. This paper presents an analytical approach for estimation of the impact of electrical vehicle (EV) battery charging on the distribution grid. Based on the EV charge profile, load curve and local distributed generation the grid nodes, the time variation of grid parameters is obtained. A set of typical load profiles of EV charging modes is studied and presented. A software implementation and a $24 \mathrm{~h}$ case study of low voltage distribution network with EV charging devices is presented in order to illustrate the approach and the impacts of EV charging on the grid. In the current paper an approach using variable nonlinear algebraic equations for dynamic time domain analysis of the charge of the electric vehicles is presented. Based on the results, the challenges due to EV charging in distribution networks including renewable energy sources are discussed. This approach is widely applicable for various EV charging and distributed energy resources studies considering control algorithms, grid stability analysis, smart grid power management and other power system analysis problems.
\end{abstract}

\section{Introduction}

In order to preserve the environment through a reduction of harmful emissions, in our days the usage of the electric vehicles is growing. And thus it is established that their charge stations are a major challenge for low voltage distribution networks stability $[1,2]$. The simultaneous operation of multiple EV charge stations represents a significant load for which most of the distribution grids are not designed for. For this reason, the influence of various EV charge profiles on microgrids is a topic of interest [3]. In the case of unidirectional charging devices (the ones that only consume power and do not provide grid support) the charging process can be represented as a distributed load to reduce grid congestion. The growing number of EV leads to a considerable increase of the load in distribution networks and difficulties in managing overloads and dispatching of the grid.

A novel approach for charging stations control in order to decrease peak loads and optimize energy flows in distribution networks is presented in this paper. Objective of the proposed solution is the implementation of a schedule which supports the grid at peak load periods using the energy storage elements of electric vehicles. This can be done by using bidirectional charging devices for EV - in this way they can consume energy in periods of excess production and supply it to the local grid afterwards. As a result, balancing of the daily energy flows can be achieved.

\section{Description of the studied microgrid}

Recently a variety of techniques and methodologies for power system analysis exist. Even in a system with multiple branches, the classical analysis of energy flows can provide a precise solution to the given problem, at the cost of increased computational time. Nevertheless limitations based on the availability of the electric vehicles are imposed - their usage is based on different repetitive patterns in weekdays and the weekend.

In the current paper an approach using variable nonlinear algebraic equations for dynamic time domain analysis of the charge of the electric vehicles is presented. On Fig. 1 is shown the studied system: a threephase $0,4 \mathrm{kV}$ distribution network with 21 nodes located in a small village near the city of Sofia [4]. The grid is equally distributed, the loads are considered symmetrical in all phases and with power factor correction (predominantly active load) therefore the compensation of reactive power is not taken into consideration in this study. The minimal and the maximal load of the system without EV charging are respectively $P_{\text {min }}=33,2 \mathrm{~kW}$ and $P_{\max }=110,8 \mathrm{~kW}$.

* Corresponding author: gergana vacheva@,tu-sofia.bg 
At node 10 and 18 of the grid are connected two photovoltaic (PV) arrays: one with a peak power $P G 1=$ $30 \mathrm{~kW}$ connected at node 10 and the second is connected at node 18 and has a peak power $P G 2=50 \mathrm{~kW}$.

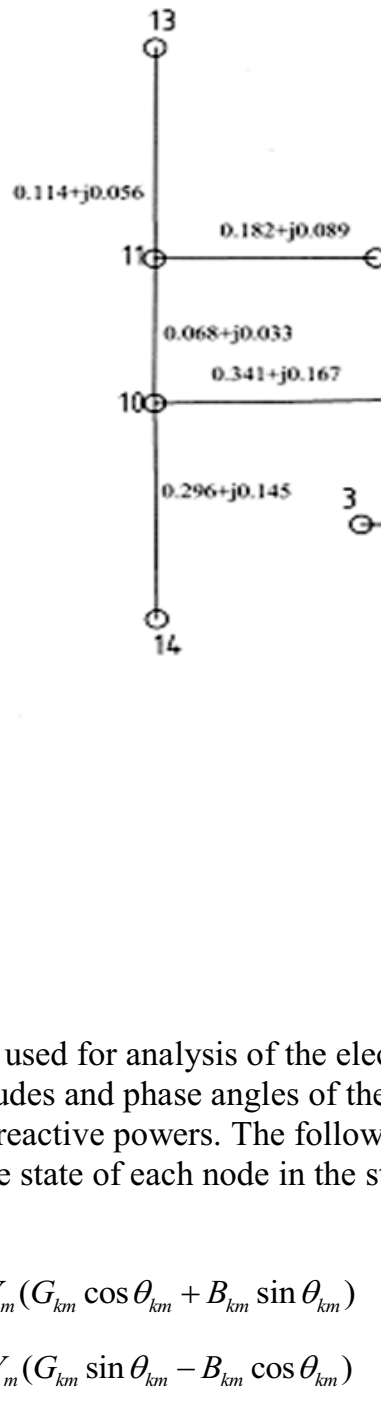

The values used for analysis of the electrical system are the magnitudes and phase angles of the voltages, the active and reactive powers. The following equations describe the state of each node in the studied microgrid [3].

$$
\begin{aligned}
& P_{k}=\sum_{m \in k} V_{k} V_{m}\left(G_{k m} \cos \theta_{k m}+B_{k m} \sin \theta_{k m}\right) \\
& Q_{k}=\sum_{m \in k} V_{k} V_{m}\left(G_{k m} \sin \theta_{k m}-B_{k m} \cos \theta_{k m}\right)
\end{aligned}
$$

Where

- $P k$ is the active power in node $k$;

- $Q k$ is the reactive power in node $k$;

- $V k$ is the magnitude of the voltage in node $k$;

- $V m$ is the magnitude of the voltage in node $m$;

$$
\dot{Y}_{k m}=G_{k m}+j B_{k m}
$$

is element $k, m$ from the bus admittance matrix;

- $\theta k$ voltage angle at node $k$;

- $\theta m$ voltage angle at node $m$;

and $\theta_{k m}=\theta_{k}-\theta_{m}$

\subsection{Types of electric vehicles present in the system}

For this study are considered four common models of electric vehicles: Mitsubishi i-MiEV family, Nissan Leaf, Tesla Model S and BMW i3. Those models were also chosen because of the fact that their characteristics and charging profiles (in normal charge and fast charge

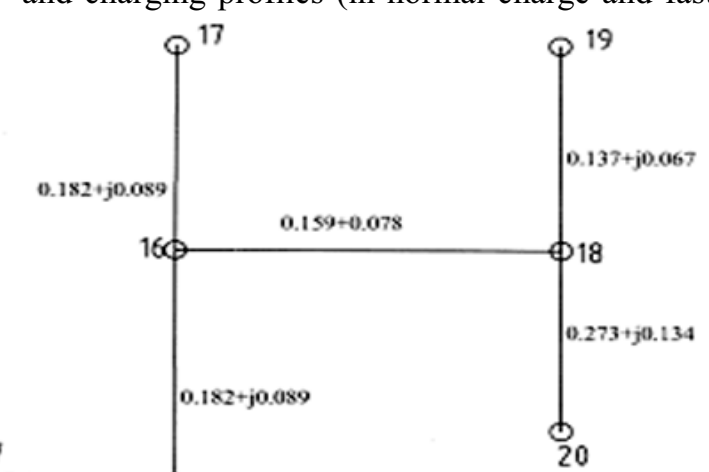

Fig. 1. One line schematic of studied microgrid

modes) are accessible from numerous sources including documentation provided by the producers. In future, the number EV in urban areas is expected to increase, each model having different charge profile, therefore the impact of an increased number of different models and charging profiles can be examined.

\subsection{Profiles of EV charging}

At the following figures (fig. 2 to fig. 10) are presented the characteristic charging profiles of the considered vehicles in different charge modes and by different values of the initial State Of Charge (SoC) - the maximum charging power is given as a function of the batteries SoC. From those characteristics and the daily grid congestion and PV production profiles, the optimal time for full charge of the vehicles is determined.

The charging stations have a significant impact on the time and mode of charging the EV batteries. The charging devices should provide relatively high instantaneous power, low cost and high efficiency of the power conversion. A classification of charging devices storage station can be made by the following parameters:

- location of the storage devices: on-board and offboard charger;

-direction of the energy flows: unidirectional and bidirectional charging devices; 
-mode of charge: normal and fast (high-power) charge.

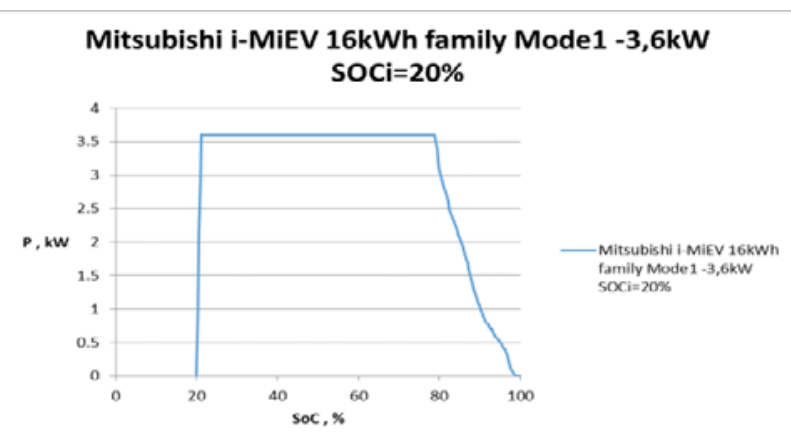

Fig. 2. State of charge profile of Mitsubishi i-Miev -3,6 kW

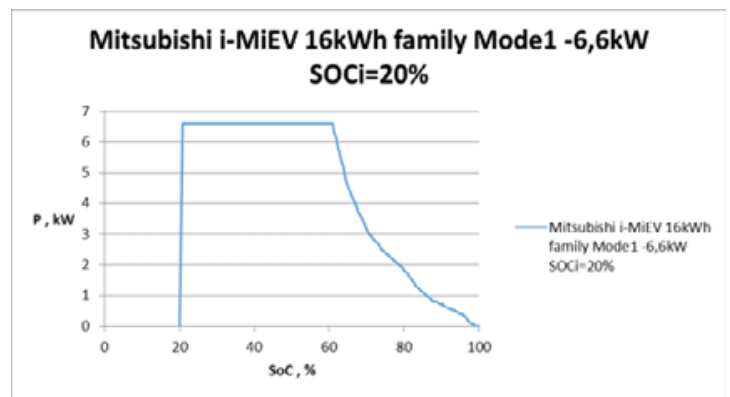

Fig. 3. State of charge profile of Mitsubishi i-Miev - 6,6 kW (fast charge)

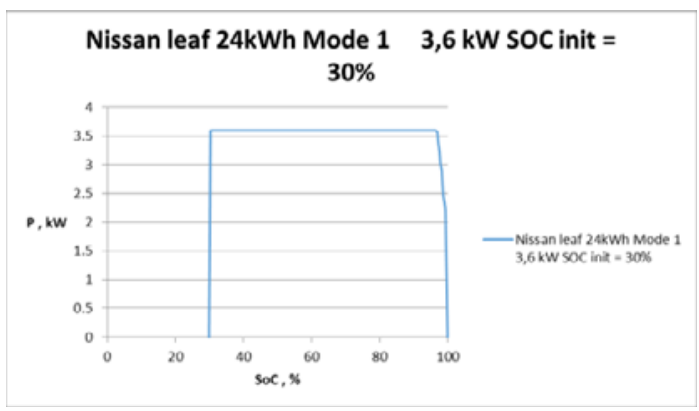

Fig. 4. State of charge of Nissan leaf - 3,6 kW

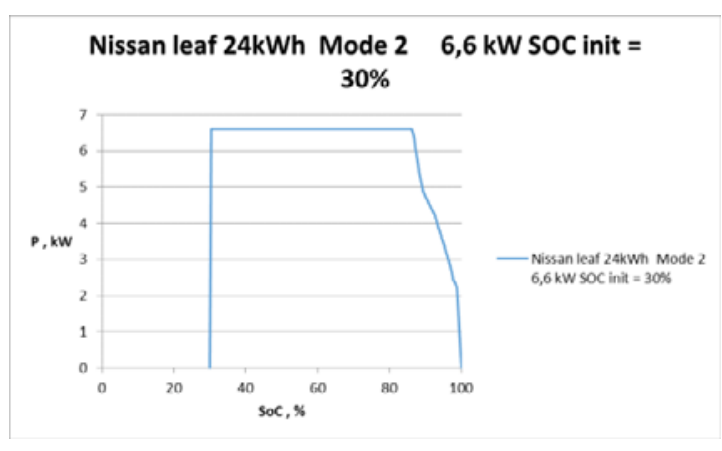

Fig. 5. State of charge of Nissan leaf - 6,6 kW (fast charge)

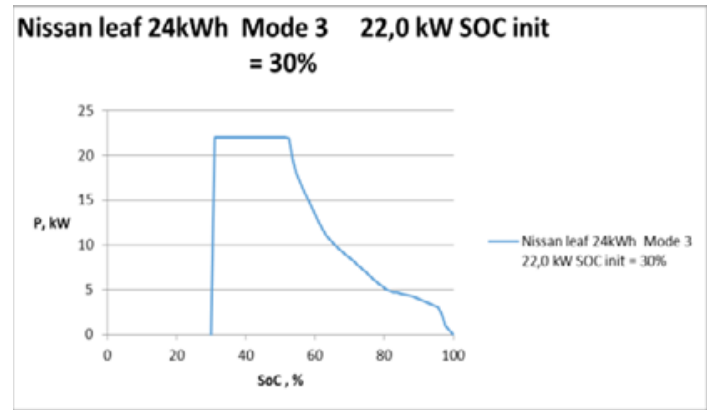

Fig. 6. State of charge of Nissan leaf - $22 \mathrm{~kW}$ (fast charge)

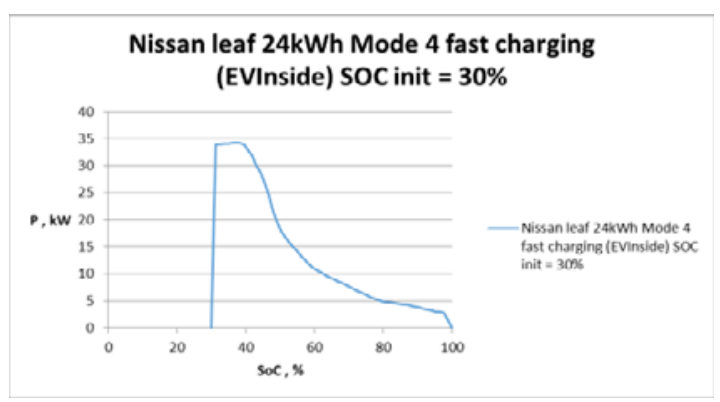

Fig. 7. State of charge of Nissan leaf - $24 \mathrm{~kW}$ (fast charge)

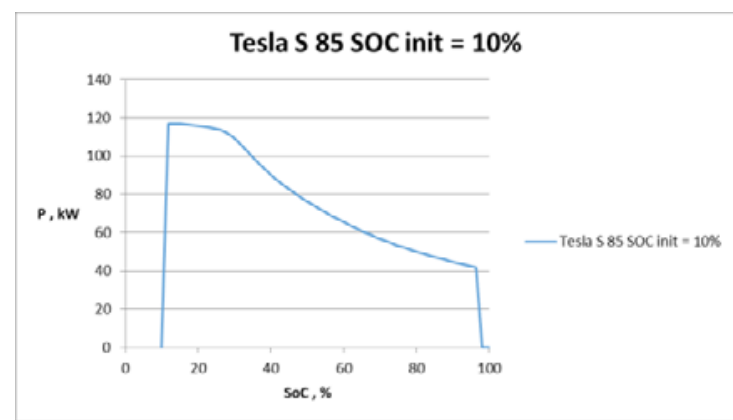

Fig. 8. State of charge of Tesla S 85 


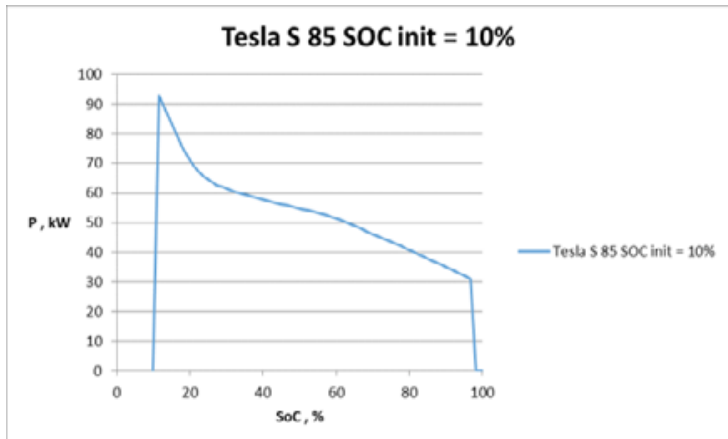

Fig. 9. State of charge of Tesla S 85 .

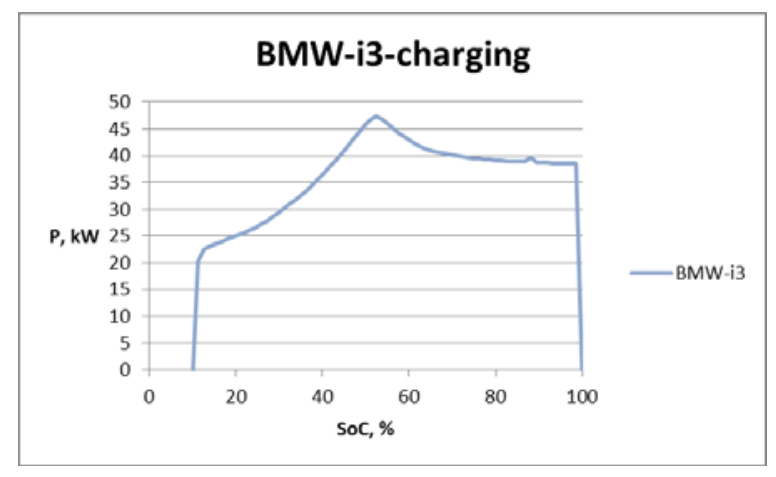

Fig. 10. State of charge of BMW i3 (fast charge)

\section{Load profile and PV generation profiles}

At the figure 11 is presented the average predictive load schedule in the studied microgrid without presence of electric vehicles for a sample 24 hour period. The forecasted power generated by the PV arrays during a sample summer day is presented on fig. 12. The data from those figures is used for assessment of the grid stability without EVs and then the impacts of EV charging in the system is assessed.

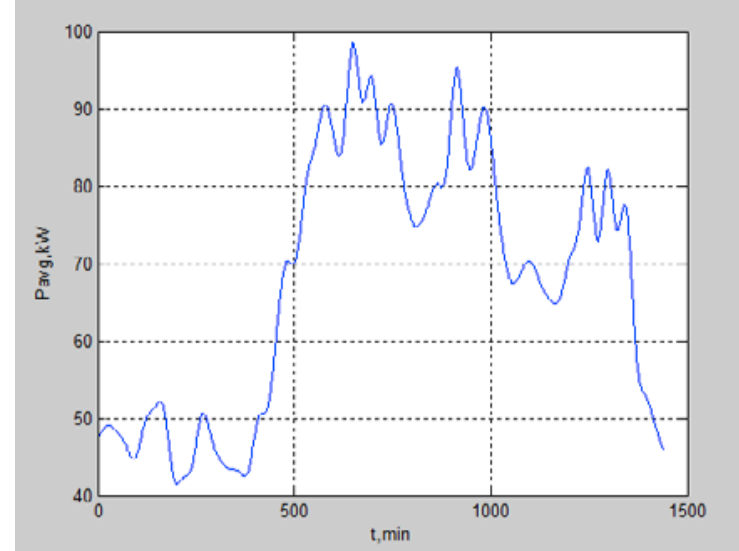

Fig. 11. Load profile forecast for a sample 24 hour period

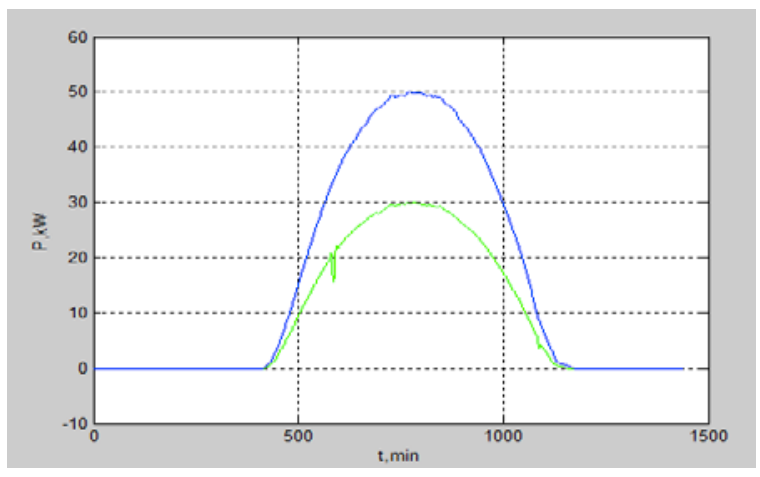

Fig. 12. PV production forecast for a sample 24 hour period

\section{Resulting load profile in the microgrid}

A presence of 10 electrical vehicles totaling $7 \%$ of the vehicles in the village is assumed. The average daily run

\begin{tabular}{|c|c|l|}
\hline Bus № & $t_{i}$, min & Charging device load pattern \\
\hline 12 & 187 & $\begin{array}{l}\text { Mitsubishi i-Mi, Mode1, } \\
\text { SOCi=20\% 3,6kW }\end{array}$ \\
\hline 14 & 300 & $\begin{array}{l}\text { Mitsubishi i-MiEV, Mode2, } \\
\text { SOCi=20\% 6,6kW }\end{array}$ \\
\hline 13 & 245 & $\begin{array}{l}\text { Mitsubishi i-Mi, Mode1, } \\
\text { SOCi=20\% 3,6kW }\end{array}$ \\
\hline 17 & 821 & $\begin{array}{l}\text { Mitsubishi i-Mi, Mode2, } \\
\text { SOCi=20\% 6,6kW }\end{array}$ \\
\hline 4 & 400 & $\begin{array}{l}\text { BMW i3, SOCi=10\%, Mode fast } \\
\text { charge }\end{array}$ \\
\hline 20 & 420 & $\begin{array}{l}\text { Nissan Leaf Mode2, SOCi=40\%, } \\
6,6 \mathrm{~kW}\end{array}$ \\
\hline 18 & 130 & $\begin{array}{l}\text { Nissan Leaf Mode3, SOCi=40\%, 22 } \\
\text { kW }\end{array}$ \\
\hline 10 & 972 & $\begin{array}{l}\text { Nissan Leaf Mode4, SOCi=40\%, } \\
\text { fast charge }\end{array}$ \\
\hline 16 & 1115 & $\begin{array}{l}\text { Tesla S 60, SOCi=10\%, Mode fast } \\
\text { charge }\end{array}$ \\
\hline 8 & 800 & $\begin{array}{l}\text { Tesla S 85, SOCi=10\%, Mode fast } \\
\text { charge }\end{array}$ \\
\hline
\end{tabular}

per vehicle is $64 \mathrm{~km}$. A distribution of the nodes of EV 
charger placement, starting moment of the charging and the initial SOC are presented in table 1. It is supposed that the most of the EV users will start charging their vehicles after returning home which is considered to occur most probably at $18: 30 \mathrm{~h}$. The resulting load profile of the grid (fig. 13) contains several peaks of the load due to EV charging, although the grid parameters remain in the operational limits, imposed by the operator.

Table 1. The scenario of the operation mode

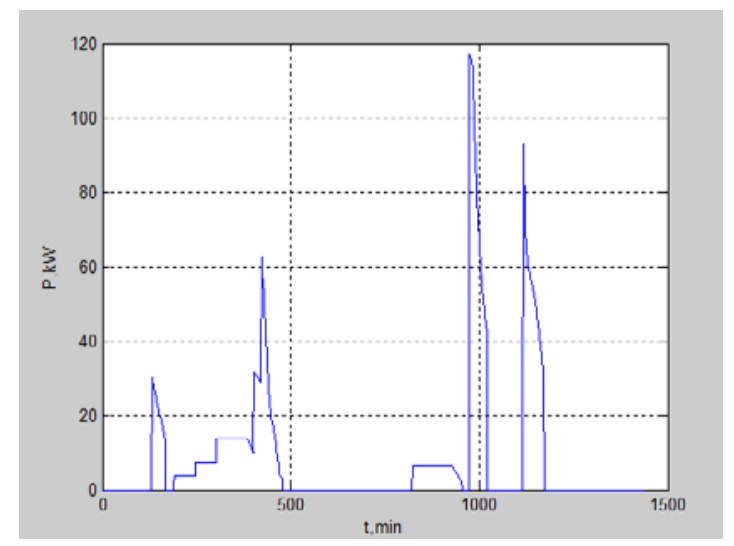

Fig. 13. Resulting load profile for the sample 24 hour period, including EV charging

\section{Conclusion}

An analytical approach for assessment of the impact of electrical vehicle battery charging on the distribution grid. Based on various EV charge profiles, a sample load curve and PV generators in various nodes of the grid, the time variation of grid parameters is obtained. A set of typical load profiles of EV charging modes is studied and presented. A software implementation and a $24 \mathrm{~h}$ case study of low voltage distribution network with EV charging devices demonstrate the impacts of EV charging on the low-voltage grid. Based on these results, the interest for future researches on bidirectional EV charging stations providing grid support in the daily periods of peak consumption is proven.

This research is funded in the framework of project BG05M2OP001-2.009-0033 of the EU Structural Funds and „Gestion intelligente des flux énergétiques dans des micro- et nano-réseaux" funded by Agence Universitaire de la Francophonie and the Bulgarian National Fund for Scientific Research.

\section{References}

1. P. Papadopoulos, "Integration of electric vehicles intro distribution Networks," Ph.D. thesis, Cardiff University, 2012.

2. Yijia Cao, Shengwei Tang, Canbing Li, Peng Zhang, et al., "An optimized EV charging model considering TOU price and SOC curve", IEEE Transactions on smart grid, Vol. 3, NO. 1, p. 388393, March 2012

3. R. Stanev, M. Georgiev, A. Krusteva, “An approach for estimation of the impact of electric vehicle charging devices on distribution networks", VI-th Scientific Conference „EF 2014“, Sozopol, Bulgaria, pp. 251-260, 22 - 25 September, 2014

4. R. Stanev, "Voltage control strategies for distribution networks with distributed energy resources", Proceedings of the Technical University of Sofia, Vol. 63, Issue 6, pp. 263-271, 2013

5. A. G. Neagoe-Stefana, A. C. Neagoe, and Al. C. Mandis, "Impact of Charging Electric Vehicles in Residential Grid on the Power Losses and Voltage Plan", International Symposium on Fundamentals of Electrical Engineering, Polytechnical University of Bucharest, Romania, November 28-28, 2014

6. S. Schey, D. Scoffield, J. Smart, "A First Look at the Impact of Electric Vehicle Charging on the Electric Grid in The EV Project", EVS26 International Battery, Hybrid and Fuel Cell Electric Vehicle Symposium, Los Angeles, California, May 6-9, 2012

7. S. Habib, M. Kamran, U. Rashid, "Impact analysis of vehicle-to-grid technology and charging strategies of electric vehicles on distribution networks - A review", Journal of Power Sources, vol. 277 pp. 205-214, March 2015

8. J. Balcells, J. Garcia, "Impact of plug-in Electric Vehicles on the supply grid", IEEE Vehicle Power and Propulsion Conference (VPPC), Lille, France, 1-3 September, 2010 